\title{
Performance of a nitrogen implanted large aperture THz emitter
}

\author{
Krzysztof Świtkowski ${ }^{*}$, Cezariusz Jastrzębski ${ }^{1}$, Norbert Pałka ${ }^{2}$, Jan Dyczewski ${ }^{3}$, Adam Barcz ${ }^{3}$ \\ ${ }^{1}$ Faculty of Physics, Warsaw University of Technology, Koszykowa 75, 00-662 Warszawa, \\ ${ }^{2}$ Institute of Optoelectronics, Military University of Technology, Kaliskiego 2, 00-908 Warszawa, \\ ${ }^{3}$ Institute of Electron Technology, Al. Lotników 32/46, 02-668 Warszawa
}

Received January 31, 2012; accepted February 07, 2012; published March 31, 2012

\begin{abstract}
Improved bandwidth of a large aperture nitrogen implanted $\mathrm{GaAs}$ photoconductive $\mathrm{THz}$ emitter is presented in this paper. An effect of nitrogen ion implantation on semi-insulating GaAs has been studied in a sample implanted at two doses: $3 \times 10^{14}$ and $6 \times 10^{14}$ ions $/ \mathrm{cm}^{2}$ The carrier life time of the investigated material was measured by means of the femtosecond time-resolved-reflectance technique. The implantation process reduced the carrier lifetime of GaAs by $65 \%$. The bandwidth of the $\mathrm{THz}$ emitter was tested in a Time Domain Spectroscopy arrangement. Usable bandwidth of the TDS set-up based on the implanted emitter increased from 1 to $1.5 \mathrm{THz}$.
\end{abstract}

$\mathrm{THz}$ radiation $(0.1 \div 10 \mathrm{THz})$ is a part of the electromagnetic wave spectrum, which has received much interest in recent years due to very promising scientific and commercial applications, especially in military, medicine and material science. Sensitive sensing of explosives which have many discrete characteristic absorption bands in the $\mathrm{THz}$ region seems to be one of the most interesting applications.

$\mathrm{THz}$ radiation has very low photon energy, which is in the range of $3 \mathrm{meV}$, and it makes $\mathrm{THz}$ radiation totally non-invasive for living cells. Therefore, $\mathrm{THz}$ radiation is ideal for people screening and medical imaging. In those fields, terahertz technique can replace X-rays, mainly in cases where spatial resolution is not critical.

The advent of femtosecond lasers has put a new perspective on coherent $\mathrm{THz}$ generation and detection. One of the most popular methods of $\mathrm{THz}$ ray generation relies on ultrafast photoconductivity. However, a short carrier lifetime semiconductor is required to emit $\mathrm{THz}$ waves in a higher frequency range. Nowadays, two such semiconductor materials are most often applied: semiinsulating (SI) GaAs and Low Temperature Grown (LTG) GaAs [1]. Still, there is a need for research in alternative superior materials. SI GaAs has quite a long carrier lifetime. LTG GaAs is a much better material for large bandwidth detection and emission of $\mathrm{THz}$ pulses. LTG GaAs epitaxial layer is grown on GaAs at a lowered substrate temperature (often below $300^{\circ} \mathrm{C}$ ) and postgrown thermally processed to obtain a short carrier lifetime and high electrical resistivity. The cost of high vacuum MBE processing is a major drawback of LTG GaAs in comparison to bare SI GaAs. LTG GaAs also

*E-mail: krzychu1@if.pw.edu.pl suffers from a problem with parameters repeatability. Recombination dynamics and electrical properties are the most problematic parameters to control in LTG GaAs. The post grown annealing is a very crucial process for optimal parameters. The best LTG GaAs material is obtained by means of rapid thermal annealing (RTA). A typical RTA process for LTG GaAs takes 30 s at $600^{\circ} \mathrm{C}$ [1]. During this step, the sample must be either in arsenide over pressure or at least covered by another GaAs wafer, in order to limit arsenide sublimation from the epitaxial layer. This process needs precise control of a temporal profile of temperature.

An alternative method to obtain a highly defected short carrier lifetime material for $\mathrm{THz}$ emitters and receivers is particle bombarding: neutron [2], proton irradiation, and ion implantation [3-4] to name a few.

Ion implantation is a desirable method because it is very well known, common and reliable process in the semiconductor industry. For the purpose of this paper, we investigated nitrogen implanted semi-insulating GaAs. Nitrogen implanted GaAs is a very interesting material.

Recently diluted nitrogen alloy $\mathrm{GaAs}_{1-\mathrm{x}} \mathrm{N}_{\mathrm{x}}$ was obtained by nitrogen implantation of GaAs. $\mathrm{GaAs}_{1-\mathrm{x}} \mathrm{N}_{\mathrm{x}}$ has very interesting optoelectronic properties like the anomalously strong band gap bowing [5] and moderate resistivity [6]. Nitrogen implanted n-GaAs was used for making ultrafast photodetectors which displayed same temporal resolution as the photodetectors made of LTG GaAs but with superior electrical properties [7]. Finally, $\mathrm{THz}$ photomixers made of nitrogen implanted n-GaAs generated higher $\mathrm{THz}$ output power with better responsivity and cut-off frequency than LTG GaAs [4].

The investigated sample was manufactured of $450 \mu \mathrm{m}$ thick vertical gradient freeze (VGF) SI GaAs wafer purchased from AXT. The sample consisted of a wafer fragment $8 \times 10 \mathrm{~mm}$. Two $3.5 \mathrm{~mm}$ wide parallel gold strip ohmic contacts were prepared on the surface of two longer sides by standard evaporation processes. On the center part of the sample, there was a $3 \mathrm{~mm}$ wide rectangular gap of uncovered GaAs. This configuration is known in the literature as a large aperture photoconductive $\mathrm{THz}$ emitter [8-9]. A large aperture emitter can be biased by much higher voltages and can be 
irradiated by a larger laser beam with much higher total power than in the case of microdipole antennae. Large aperture configuration gives high $\mathrm{THz}$ power but at the expense of $\mathrm{THz}$ emission bandwidth.

The sample was nitrogen implanted in a pelletron accelerator (National Electrostatics Corporation model 3SDH-2). The cathode with compound TiN and graphite was used as a source of negative ions by cesium sputtering. Produced $\mathrm{CN}^{-}$ions are accelerated and broke into carbon and nitrogen ions. Nitrogen ions are selected by magnets and finally accelerated to energy of $800 \mathrm{keV}$.

Figure 1 shows the Monte Carlo simulation of an implantation profile. For this purpose we used the computer program TRIM [10]. The maximum nitrogen concentration is around $1 \mu \mathrm{m}$ beneath the sample surface.

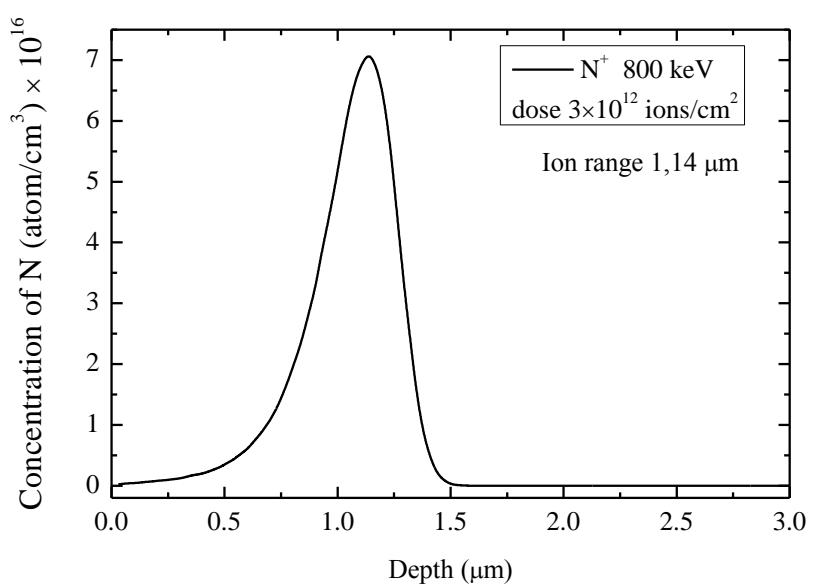

Fig.1. TRIM-simulated profile of the penetration depth of implanted $\mathrm{N}^{+}$ ions into GaAs.

The carrier lifetime was measured in time resolved reflectivity pump and probe configuration [11]. Sample was excited and probed by a Ti:Sapphire mode-locked laser. The laser produced a pulse train at $80 \mathrm{MHz}$ repetition rate and a pulse width of $100 \mathrm{fs}$.

All measurements were carried out at laser beam excitation average power of $3 \mathrm{~mW}$. Reflectivity was probed by a variablytime delayed beam. The average power of probing laser beam was about $150 \mu \mathrm{W}$. Both beams were focused to about $10 \mu \mathrm{m}$ and spatially overlapped on the sample surface.

Figure 2 shows the temporal evolution of relative reflectivity change of the sample before implantation versusthe same sample after ion implantation at dose $3 \times 10^{12}$ ions $/ \mathrm{cm}^{2}$ and after another subsequent implantation at the same dose.

All curves were fitted by means of the double exponential decay function.

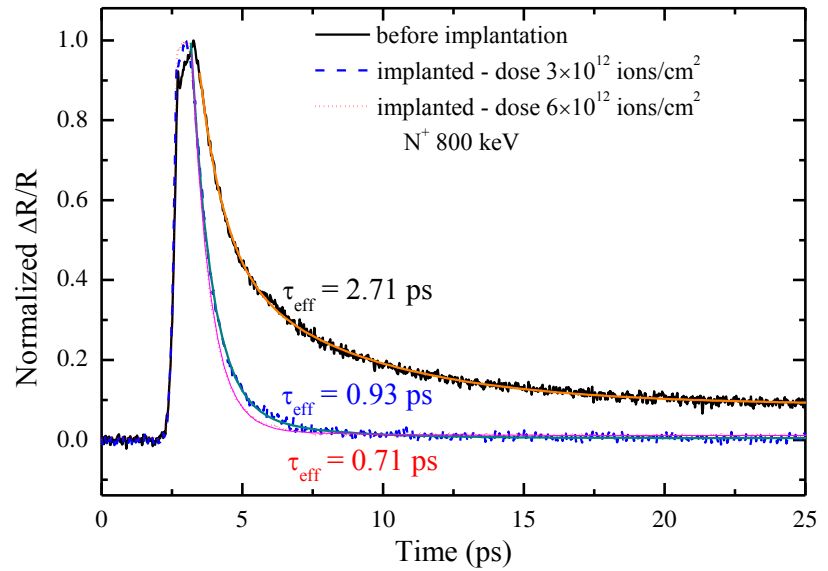

Fig.2. Time resolved reflectivity change for implanted sample at two doses versus non implanted.

An effective carrier lifetime was calculated from the fit parameters according to:

$$
\tau_{\text {eff }}=\frac{A_{1} \tau_{1}+A_{2} \tau_{2}}{A_{1}+A_{2}}
$$

where $A_{1}$ and $A_{2}$, and $\tau_{1}$ and $\tau_{2}$ are the amplitudes, and decay parameters of exponential functions, respectively.

Nitrogen implantation reduced the effective carrier lifetime from 2.71 to 0.93 . After adding another dose, the carrier lifetime reduction was much smaller. From our preliminary studies, we can state that the dose $3 \times 10^{12}$ ions/cm is close to optimal for the $\mathrm{THz}$ emitter. Furthermore, we noticed a dramatic sample resistance drop from $330 \mathrm{M} \Omega$ to $27.3 \mathrm{M} \Omega$ for the material implanted at dose $3 \times 10^{12}$ ions $/ \mathrm{cm}$ and implanted at $6 \times 10^{12}$ ions $/ \mathrm{cm}$, respectively.

The performance of the sample operating as the $\mathrm{THz}$ emitter was verified by means of the Time Domain Spectroscopy technique (TDS). The TDS setup was based on a commercial TeraVil Ltd. LTG GaAs detector. The detector has a silicon $\mathrm{THz}$ collimating lens. The implanted sample was applied as a $\mathrm{THz}$ dc biased emitter. The emitter was positioned very close to the detector and no extra $\mathrm{THz}$ optics was used from the side of the emitter. The bias voltage was provided from a current controlled high voltage source. The voltage source was set to $100 \mathrm{~V}$. The current was limited to $20 \mathrm{~mA}$. The femtosecond laser beam for the emitter excitation was expanded to a diameter of $3 \mathrm{~mm}$, and had an average power of $600 \mathrm{~mW}$. The obtained electric field waveforms from the detector were normalized for better comparison of $\mathrm{THz}$ pulse widths (Fig.3).

The implantation decreased pulse full width at half maximum (FWHM) from $1.2 \mathrm{ps}$ to $0.71 \mathrm{ps}$ for unimplanted and implanted structure, respectively.

The observed trend agrees with our carrier lifetime measurements (see Fig. 2). 


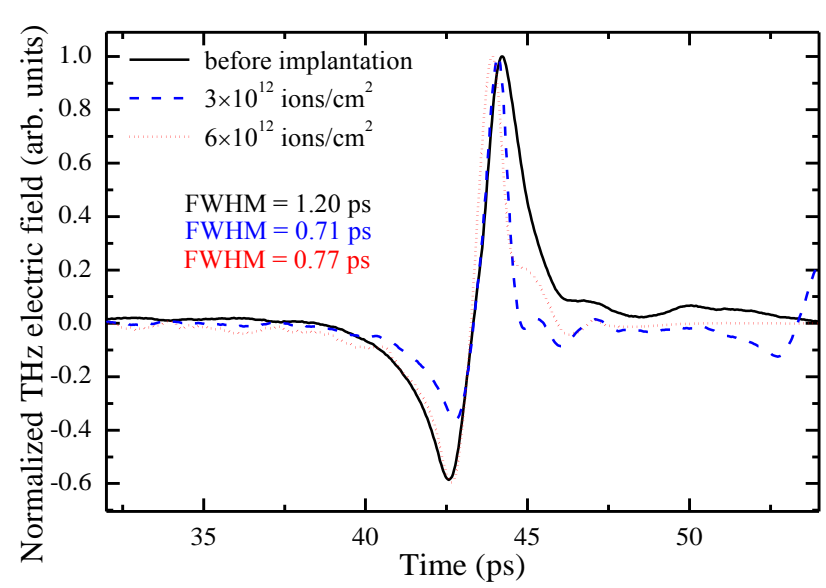

Fig.3. Measured THz wave forms for unimplanted, implanted and double implanted sample.

Adding another dose did not cause an improvement in $\mathrm{THz}$ pulse duration (Fig.3). Moreover, we observed that the amplitude of a $\mathrm{THz}$ pulse after the first implantation decreased twofold. After the second implantation, the amplitude decreased eight times in respect to the unimplanted sample.

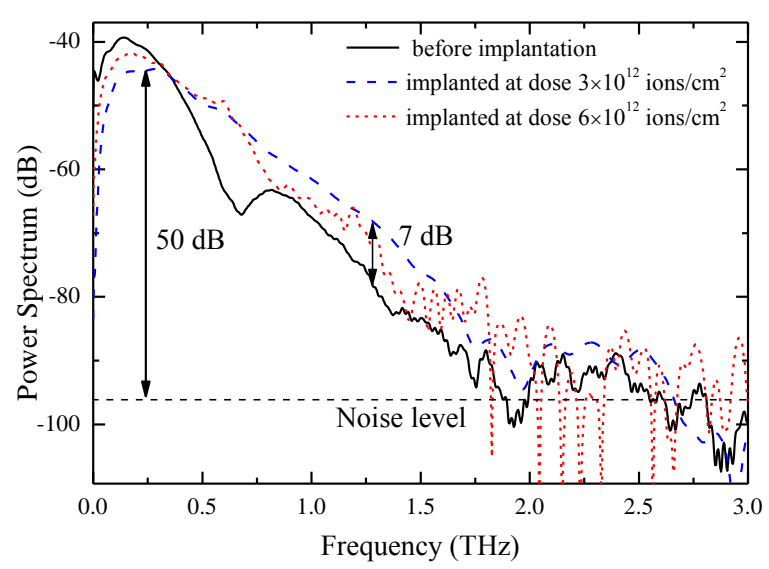

Fig.4. Electric THz field spectrum for unimplanted, implanted and double implanted samples.
The comparison of the FFT power spectrum for normalized time domain $\mathrm{THz}$ pulses of an implanted structure with unimplanted, revealed a substantial power increase at $650 \mathrm{GHz}$ and about $7 \mathrm{~dB}$ power gain in the range up to $1.6 \mathrm{THz}$ (Fig.4). Further implantation did not improve the power spectrum. However, the emitter implanted at a higher dose gave a weaker signal and a much lower signal to noise ratio than a single implanted one.

Nitrogen implantation of GaAs at dose $3 \times 10^{12}$ ions $/ \mathrm{cm}^{2}$ is advantageous for a more broadband $\mathrm{THz}$ emitter. A further dose increase does not give any benefits in the $\mathrm{THz}$ emitter performance.

To sum up, we studied the influence of Nitrogen implantation of a semi-insulating GaAs emitter on its temporal and spectral characteristics in the TDS set-up. For the implantation dose $3 \times 10^{12}$ ions $/ \mathrm{cm}^{2}$ we obtained the duration of the $\mathrm{THz}$ impulse at FWHM of $0.71 \mathrm{ps}$. The width of the corresponding spectrum is usable in the $0.1 \div 1.6 \mathrm{THz}$ range and the dynamic range is equal to $50 \mathrm{~dB}$ at maximum.

This work has been supported by the European Union in the framework of European Social Fund through the Warsaw University of Technology Development Programme, realized by the Center for Advanced Studies.

\section{References}

[1] M.R. Melloch et al., Annu. Rev. Mater. Sci. 25, 547 (1995).

[2] C. Wang, M. Pocha, J. Morse, M. Singh, B. Davis, Appl. Phys. Lett. 54, 1451 (1989).

[3] H.H. Tan et al., IEEE J. Sel. Top. Quantum Electron. 2, 636 (1996).

[4] P. Kordos, M. Marso, and M. Miculics, Appl. Phys. A, 87563 (2007).

[5] W. Shan, K.M. Yu, W. Walukiewicz, J.W. Ager III, E.E. Haller, and M.C. Ridgeway, Appl. Phys. Lett. 75, 1410 (1999).

[6] A. Patanè, G. Allison, L. Eaves, M. Hopkinson, G. Hill, and A. Ignatov, J. Phys. Condens. Matter 21, 174209 (2009).

[7] M. Mikulics, M. Marso, P. Kordo, S. Stanek, P. Ková, X. Zheng, S. Wu, and R. Sobolewski, Appl. Phys. Lett. 83, 1719 (2003).

[8] A.S. Weiling, B.B. Hu, N. M. Froberg, and D.H. Auston, Appl. Phys. Lett. 64, 137 (1994).

[9] S.G. Park, A.M. Weiner, M.R. Melloch, C.W. Siders, J.L.W. Seiders, and A.J. Taylor, IEEE J. Quantum Electron. 35, 1257 (1999).

[10] F. Ziegler, J.P. Biersack and U. Littmark, The Stopping and Range of Ions in Solids (New York, Pergamon 1985).

[11] C.V. Shank et al., Solid State Commun. 26, 567 (1978). 